\title{
Label-free real-time optical monitoring of DNA hybridization using SiN Mach-Zehnder interferometer-based integrated biosensing platform
}

Mohammed Sharif Murib

Daan Martens

Peter Bienstman 


\title{
Label-free real-time optical monitoring of DNA hybridization using SiN Mach-Zehnder interferometer-based integrated biosensing platform
}

\author{
Mohammed Sharif Murib, ${ }^{\mathrm{a}, \mathrm{b}}$ Daan Martens, ${ }^{\mathrm{a}, \mathrm{b}}$ and Peter Bienstman ${ }^{\mathrm{a}, \mathrm{b}, *}$ \\ ${ }^{a}$ Ghent University/Imec, Photonics Research Group, Ghent, Belgium \\ ${ }^{\mathrm{b}}$ Ghent University, Center for Nano- and Biophotonics (NB-Photonics), Ghent, Belgium
}

\begin{abstract}
We report on the label-free real-time optical monitoring of DNA hybridization upon exposure to a flow of complementary DNA at different concentrations. The biosensor is composed of a silicon nitride integrated unbalanced Mach-Zehnder interferometer (MZI), with an integrated arrayed waveguide grating as a spectral filter. This MZI has been shown to have both sufficient multiplexing capability and limit of detection on the order of $10^{-6}$ RIU. Probe DNA, consisting of a 36-mer fragment is covalently immobilized on the silicon nitride integrated biosensor. The wavelength shift is monitored upon complementary DNA targets being flown over the sensor. Concentrations of $1 \mathrm{pM}$ can be easily detected. Also, an alternative route to modify the sensor surface with carboxylic groups using the photochemical reaction of fatty acids is proposed and preliminary XPS results are presented. Moreover, preliminary results for DNA obtained from a rolling circle amplification (RCA-DNA) process and spiked in a realistic amplification buffer are presented. ๑ 2018 Society of Photo-Optical Instrumentation Engineers (SPIE) [DOI: 10.1117/1.JBO.23.12.127002]
\end{abstract}

Keywords: label-free biosensor; Mach-Zehnder interferometer; DNA; silicon nitride.

Paper 180278R received May 15, 2018; accepted for publication Dec. 3, 2018; published online Dec. 21, 2018.

\section{Introduction}

There is a growing demand for sensors to detect nanoparticles, viruses, ${ }^{1}$ and biomolecules ${ }^{1}$ because of recent advances in nanotechnology and biology. In particular, clinical diagnostics demands sensitive real-time- and label-free detection techniques. $^{2}$ Biomolecule recognition and deoxyribonucleic acid (DNA) denaturation were studied by different types of measurement setups, such as microfluidic sensing, ${ }^{3}$ elastic measurements at the level of a single molecule, ${ }^{4}$ force-induced denaturation, ${ }^{5}$ and by monitoring the denaturation dynamics in real time using impedance spectroscopy. ${ }^{6}$ Other label-free biosensors that have been shown recently are nanowires, ${ }^{7}$ nanoparticle probes,${ }^{8}$ biochips, ${ }^{9}$ mechanical cantilevers, ${ }^{10}$ and field-effect sensors. ${ }^{11,12}$ Label-free optical biosensors offer great advantages over more conventional analytical techniques. ${ }^{13,14}$ This is related to the fact that optical biosensors are highly sensitive, fast, reproducible, and circumvent the need to modify target molecules by labeling. ${ }^{2,13,14}$ Optical biosensors are powerful transducers that can detect the presence of (bio)chemical molecules at a surface as well as physical properties of the medium. ${ }^{15,16}$ For example, in their seminal work, Arnold et al. ${ }^{17,18}$ and M. A. Santiago-Cordoba et al. ${ }^{19}$ demonstrated protein adsorption in spherical photonic microcavities. Zlatanovic et al. ${ }^{2}$ demonstrated the detection of the presence and concentration of proteins in a physiological buffer using photonic crystal microcavities. Optical biosensors are insensitive to electromagnetic interference, capable of performing remote sensing, and can provide multiplexed detection within a single device. ${ }^{20}$ Also, optical biosensors can be extremely sensitive (nanomolar

*Address all correspondence to Peter Bienstman, E-mail: peter.bienstman@ ugent.be concentrations or less), nondestructive to the sample, and the transduction processes in optical biosensors generally take place at a surface and can be tailored to sense almost any kind of molecule, chemical and biological. ${ }^{21}$

Optical sensing can be performed using ring resonators, ${ }^{22}$ confocal microscopy, ${ }^{6}$ prism couplers, ${ }^{23}$ spherical cavities, ${ }^{24}$ and fiber-optic waveguides. ${ }^{25,26}$ In ring resonators, spherical cavities, and fiber-optics waveguides, the light is coupled through the waveguides, and an evanescent field extends beyond the waveguide surface by $\approx 100 \mathrm{~nm}^{21}$ The analytes bound to the surfaces of these waveguides will lie in the path of the evanescent field, and as a result, they change the effective size and refractive index of the guided mode. ${ }^{21}$ Optical sensing using confocal microscopy and fiber-optic waveguides has certain disadvantages. For example, fiber-optics waveguide sensors are typically quite long. To achieve a high signal and low detection limit, the waveguide must be on the order of a few centimeters long, because the sensing signal is accumulated along the waveguide. ${ }^{21}$ On the other hand, some other fiber-optics-based biosensors can be quite sensitive label-free biosensors, but the whole system is more difficult to miniaturize further, compared with chip-based sensors. ${ }^{27}$ Confocal microscopy requires fluorescent labeling for detection, which is time-consuming, complex, and may not always be suitable for rapid biophysical and routine characterization tasks. ${ }^{28}$ In addition, real-time hybridization monitoring using confocal microscopy cannot be performed, because it is hard to distinguish between unbound targets in solution and those that have been hybridized to the probe on the surface, as both will fluoresce when imaged. ${ }^{29}$ Photonic microcavities such as circular or spherical resonators 
are promising optical label-free detection setups. ${ }^{23,30}$ In a photonic microcavity, the target molecules are sampled hundreds of times because of the recirculation of light within the microcavity by total internal reflection. ${ }^{28}$ The target molecules induce a change in the optical microcavity properties such as the size and refractive index; and as a result, a change in the whispering gallery mode resonant wavelength is encountered. ${ }^{31}$ Also, a silicon-on-insulator microring resonator for sensitive label-free biosensing was fabricated. ${ }^{32-34}$ After coating with biotin receptor molecules, a detection limit of $0.37-\mathrm{fg}$ avidin mass (3260 molecules) was shown. ${ }^{35,36}$

In this article, an experimental study of an optical biosensor is performed, which is composed of a silicon nitride $\left(\mathrm{Si}_{3} \mathrm{~N}_{4}\right)$ integrated unbalanced Mach-Zehnder interferometer (MZI) ${ }^{37}$ with integrated spectrometer. This MZI has been shown to have both sufficient multiplex capability and limit of detection on the order of $10^{-6}$. The silicon nitride sensor surface was functionalized with DNA layers, and the corresponding shift in wavelength was monitored and analyzed. Silicon nitride shows outstanding chemical inertness and robustness for protecting biosensing devices from solutions that would otherwise render them inoperable. ${ }^{38}$ Moreover, with respect to an extensive comparison between different sensor types, ${ }^{39}$ the main advantage of our sensor is performance, which is very close to the state of the art, while being compatible with point-of-care systems.

\section{Silicon Nitride Chip Surface Modification with Double-Stranded DNA}

Probe DNA consisting of a 36-mer fragment was covalently immobilized on $\mathrm{Si}_{3} \mathrm{~N}_{4}$ chips. The DNA probe sequence used in the study was $5^{\prime} \mathrm{NH}_{2}$-TTT TTT TTT TCA CCA GCT CCA ACT ACC AC-3' and target DNA sequence was 5' GTG GTA GTT GGA GCT GAA AAA AAA AA_3'. These fragment lengths comply well with typical lengths used in the commercially available DNA microarray platforms. The $\mathrm{Si}_{3} \mathrm{~N}_{4}$ chips were cleaned in acetone, isopropanol, and ultrapure water. Next, the chips were exposed to oxygen plasma for one minute using a reactive-ion etching (RIE) system to terminate the surface with $\mathrm{OH}$ groups on the surface. Afterward, the chips were terminated with $\mathrm{NH}_{2}$ groups using a silanization route. Silanization of the $\mathrm{Si}_{3} \mathrm{~N}_{4}$ chips was carried out by the liquid phase deposition of a solution of silane in an organic solvent. ${ }^{40}$ The chips were placed in a $600-\mathrm{mM}$ solution of (3-aminopropyl) triethoxysilane (APTES, 99\%; Sigma-Aldrich, St. Louis, Missouri) in toluene ( $\geq 99.9 \%$, Sigma-Aldrich) for $15 \mathrm{~h}$ in a nitrogen-filled glovebox. To wash off the unbound APTES, the samples were first rinsed with toluene, followed by tetrahydrofuran ( $\geq 99.9 \%$, Sigma-Aldrich). After drying the samples in a nitrogen stream, they were cured for $30 \mathrm{~min}$ at $130^{\circ} \mathrm{C}$. Curing at this high temperature will create a much stronger film of APTES on the surface. ${ }^{35}$ This silanization step creates an amine-modified surface, ${ }^{41}$ allowing for the subsequent reaction with succinic anhydride (SA) to yield a carboxyl-terminated surface. Succinic anhydride (SA, $\geq 99.9 \%$, Sigma-Aldrich) was used as a cross-linker molecule to ensure the binding of the amino group of APTES to the $\mathrm{NH}_{2}$-terminated DNA strands by providing carboxylic groups at the surface. ${ }^{36,42}$ For this purpose, the chips were placed overnight in a solution of $800 \mathrm{mM}$ of SA in acetonitrile (ACN) (anhydrous, $99.8 \%$, Sigma-Aldrich), after which they were rinsed with pure acetonitrile and dried under nitrogen gas. ${ }^{37}$ The amine-terminated surface reacts with one of the two carbonyl
Table 1 XPS results of the $\mathrm{Si}_{3} \mathrm{~N}_{4}$ chips surface modified with $\mathrm{COOH}$ groups using fatty acids route.

\begin{tabular}{lcccccccc}
\hline $\mathrm{C} 1 \mathrm{~s} \%$ & $\mathrm{~N} 1 \mathrm{~s} \%$ & $\mathrm{O} 1 \mathrm{~s} \%$ & $\begin{array}{c}\mathrm{Si} 2 \mathrm{p} \\
\%\end{array}$ & $\begin{array}{c}\mathrm{C}-\mathrm{C} \\
\%\end{array}$ & $\begin{array}{c}\mathrm{C}-\mathrm{O}-\mathrm{C} \\
\%\end{array}$ & $\begin{array}{c}\mathrm{O}-\mathrm{C}=\mathrm{O} \\
\%\end{array}$ & \\
\hline 72.4 & 3.9 & 19.1 & 4.7 & 82.0 & 10.8 & 7.2 & Blank \\
69.6 & 0.4 & 25.2 & 4.8 & 50.5 & 37.7 & 11.8 & $\begin{array}{l}\text { Fatty } \\
\text { acids }\end{array}$ \\
\hline
\end{tabular}

groups of the SA molecules, causing the anhydride ring to open and to form a covalent bond with this carbonyl group. The other carbonyl group is released to form a free carboxylic acid-terminated surface. ${ }^{43}$ The zero-length cross linker, 1-ethyl3-[3-dimethylaminopropyl]-carbodiimide was used for the covalent coupling of the $5^{\prime}$ amine-modified side of a 36-mer single-stranded DNA (ssDNA) fragment to the carboxylmodified surface in 2-[N-morpholino]-ethannesulphonic acid buffer at $4^{\circ} \mathrm{C} . .^{37,44}$ Nonspecifically bound DNA was removed using multiple washing steps. In a first step, chips were washed in $1 \times$ PBS (10 min, RT). Next, the chips were washed in $2 \times \mathrm{SSC} / 0.5 \%$ SDS $(30 \mathrm{~min}, \mathrm{RT})$. Finally, they were rinsed with $1 \times$ PBS. $^{39}$

An alternative route based on fatty acids was also briefly investigated to modify the surface with $\mathrm{NH}_{2}$-terminated DNA. Such a route proved to save incubation time, chemicals, and equipment. This route was applied before for nanocrystalline diamond surface modification with carboxylic groups. ${ }^{6}$ First, the $\mathrm{Si}_{3} \mathrm{~N}_{4}$ chips were placed inside a glovebox under nitrogen atmosphere. The chips were covered with a thin film of unsaturated fatty acid (10-undecenoic acid) and were exposed to $\mathrm{UV}$ radiation $\left(254 \mathrm{~nm}, 265 \mathrm{~mW} \mathrm{~cm}^{-2}\right)$ for $3 \mathrm{~h}$ under nitrogen atmosphere. The double bonds of the unsaturated fatty acid chains were broken down and a covalent bond with the hydrogen-terminated $\mathrm{Si}_{3} \mathrm{~N}_{4}$ was established. This process was mediated by photoemmission from the surface as proposed for the photochemical grafting of alkenes to silicon surfaces. ${ }^{45,46}$ The unbound fatty acid chains were washed off using hexane. In this way, a carboxyl $(\mathrm{COOH})$-terminated $\mathrm{Si}_{3} \mathrm{~N}_{4}$ surface was obtained. Afterward, zero-length 1-ethyl-3-3-dimethylaminopropyl-carbodiimide (EDC) can be used for the covalent coupling of the $\mathrm{NH}_{2}$-terminated DNA to the carboxyl-terminated surface in 2-[N-morpholino]-ethannesulphonic acid (MES) buffer at $4^{\circ} \mathrm{C}$.

This procedure was evaluated using XPS measurements performed on planar silicon nitride chips. XPS is particularly useful for such an analysis as it can provide information about the chemical environment of specific atoms by establishing binding-energy shifts of appropriate core levels (chemical shifts). Table 1 shows the XPS results of a blank silicon nitride chip and a chip modified with a carboxylic group using the fatty acids route. The carboxylic group formed on the surface has increased by $4 \%$ as compared with the blank sample.

However, for the experiments mentioned below, we decided to focus on the APTES route, as the fatty acid route still needed to be optimized further.

\section{Photonic Detection Mechanism}

Figure 1(a) shows a schematic of the optical setup used for the DNA detection. It is composed of a silicon nitride $\left(\mathrm{Si}_{3} \mathrm{~N}_{4}\right)$ integrated unbalanced MZI, which contains an integrated spectral 


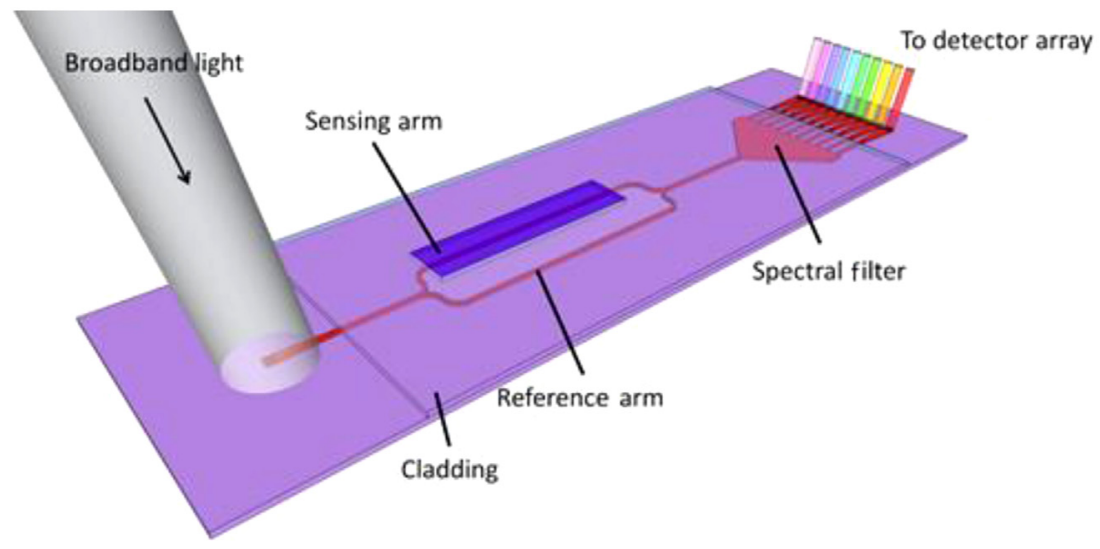

(a)

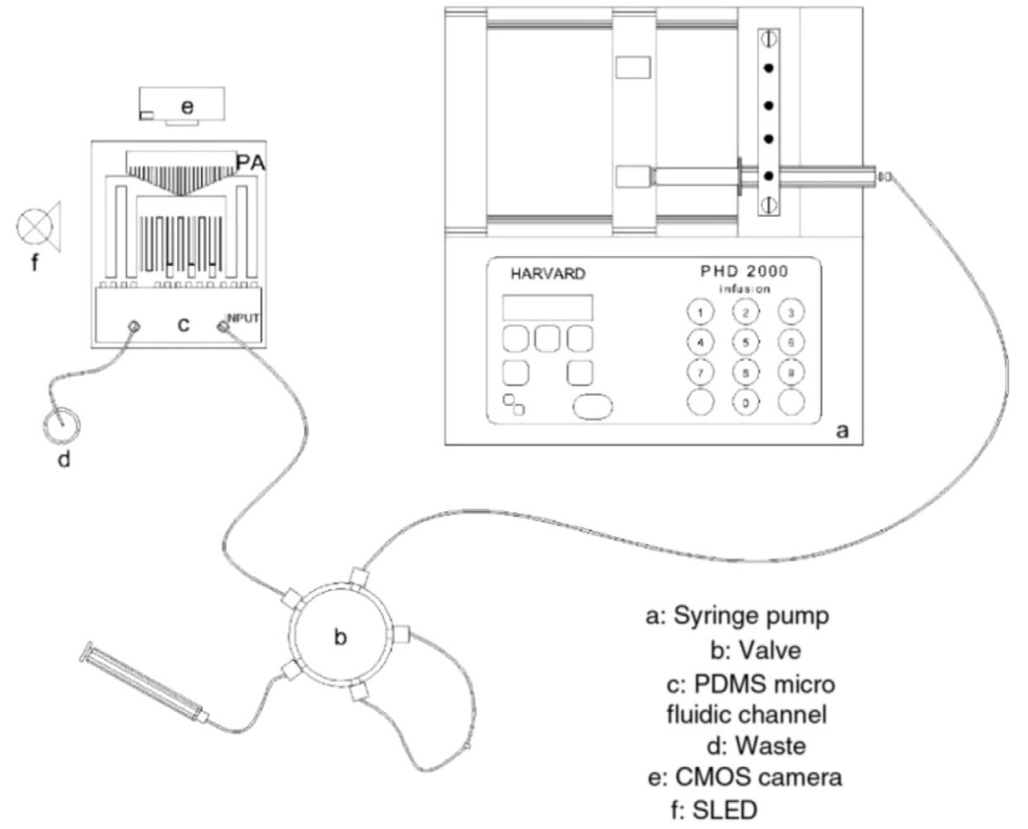

(b)

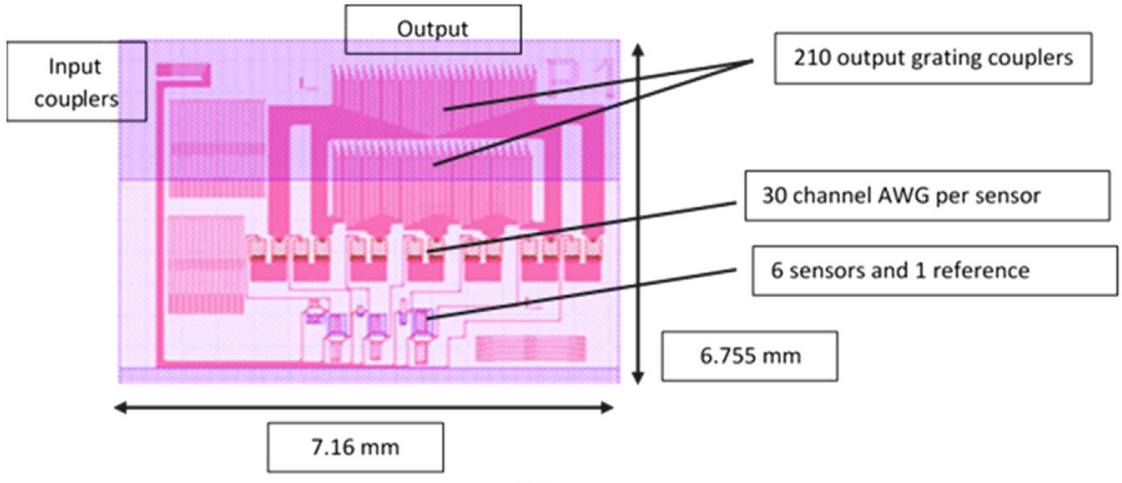

(c)

Fig. 1 (a) Schematic of the optical setup used for the DNA detection, (b) schematic of the experimental setup, and (c) photonic integrated circuits (PICs) sensor.

filter to allow for a cheap readout. ${ }^{47}$ This MZI has been shown to have both sufficient multiplex capability and limit of detection on the order of $10^{-6}$. The detection limit is in principle a function of the length of the arms of the interferometer, but also depends on other factors like the number of wavelength channels and the noise of the light source. More details can be found in Martens and Bienstman. ${ }^{48}$ An $850-\mathrm{nm}$ free space SLED broadband source is coupled into the on-chip optical circuit 
through a 16-deg vertical grating coupler. Then, the light is guided along a monomode strip waveguide. The light in the monomode strip waveguide is subsequently divided between two arms using an MMI coupler. One arm is covered with an oxide cladding layer and the other arm is exposed to the analyte. A second MMI coupler is then used to combine the light from both arms.

As a result, at the output of the second MMI, an intensity modulation is encountered depending on the phase shift caused by the analyte samples grafted over the sensing arm. The MZI output is then spectrally filtered through an on-chip arrayed waveguide grating into 30 channels with 1-nm bandwidth each before the light exits the chip. ${ }^{37}$ A CMOS camera placed vertically over the chip is used to measure the output optical intensity from the couplers. Moreover, a syringe pump [Fig. 1(b)] is used to pump PBS buffer $(\mathrm{pH}=7)$. A dualmode injector is used to load the complementary DNA samples to be tested in line with the neutral buffer. The sensor chip is squeezed under a home-mode PDMS microfluidic channel. The PDMS microfluidic channel has a height of $50 \mu \mathrm{m}$ and width of $600 \mu \mathrm{m}$.

Figure 1(c) shows the photonic-integrated circuit (PIC) sensor that was fabricated based on the CMOS compatible SiN photonics developed at imec, using wafer-level processing on standard 725- $\mu \mathrm{m}$-thick $200-\mathrm{mm} \mathrm{Si}$ wafers. ${ }^{49}$ First, a 70 -nmthick SiN antireflective coating was deposited using plasmaenhanced chemical vapor deposition (PECVD) using $\mathrm{SiH}_{4}$, $\mathrm{N}_{2}$, and $\mathrm{NH}_{3}$ at $400^{\circ} \mathrm{C}$. ${ }^{49}$ Subsequently, a $2.3-\mu \mathrm{m}$-thick PECVD $\mathrm{SiO}_{2}$ layer, which had been specifically engineered to compensate the stress level of the wafers, was deposited using $\mathrm{SiH}_{4}$ and $\mathrm{N}_{2} \mathrm{O}$. On top of this oxide layer, a 220-nmthick PECVD SiN layer was deposited using $\mathrm{SiH}_{4}, \mathrm{~N}_{2}$, and $\mathrm{NH}_{3}$ at $400^{\circ} \mathrm{C}$.

Grating couplers were defined using 193-nm deep ultraviolet (DUV) lithography followed by a shallow etch of 110-nm depth into the SiN to maximize light coupling into the waveguide. A fluorine-based inductively coupled plasma reactive ion etching process was used to minimize sidewall roughness and achieve the required sidewall slope. Next, waveguides were defined by a separate 193-nm DUV lithography step and similar etch, but now the full thickness of the SiN layer was etched. Following this process, a $2-\mu \mathrm{m}$ layer of PECVD $\mathrm{SiO}_{2}$ was deposited and planarized by CMP. Finally, sensing regions were defined by lithography using the i-line of $\mathrm{Hg}$ at $365 \mathrm{~nm}$ followed by a selective removal of the top oxide cladding using a dry etching process followed by a wet etching step. ${ }^{49}$

Sensors were initially characterized through bulk sensing in a lab-based setup, with equipment compatible with point-of-care sensing. Transmission spectrum of the proposed Mach-Zehnder interferometer and more details are described by Martens et al. ${ }^{37}$

The chips are designed to allow detection of up to six sensors in parallel. Multiplexing capability with this chips was shown by Martens et al. ${ }^{50}$

\section{Experimental Results}

A series of different of concentrations of complementary DNA and noncomplementary DNA were studied. Figures 2 and 3 show the real-time interaction of noncomplementary DNA samples and complementary DNA samples with the ssDNA molecules immobilized on the SiN sensors, respectively. First, 1× PBS (pH 7) was administered to the sensors. After stabilization of the signal, $200 \mu \mathrm{L}$ of complementary or

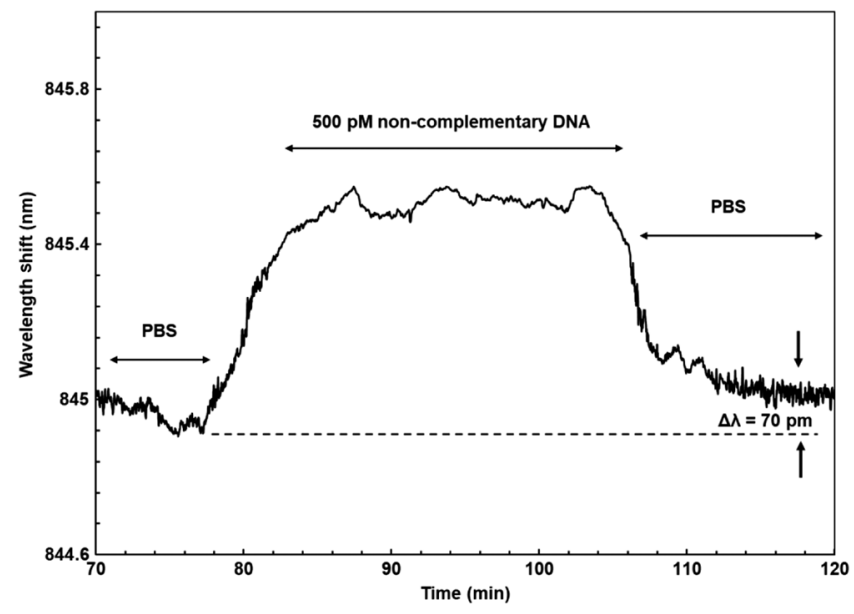

Fig. 2 Wavelength shift due flow of 500 pM noncomplementary DNA on the sensor.

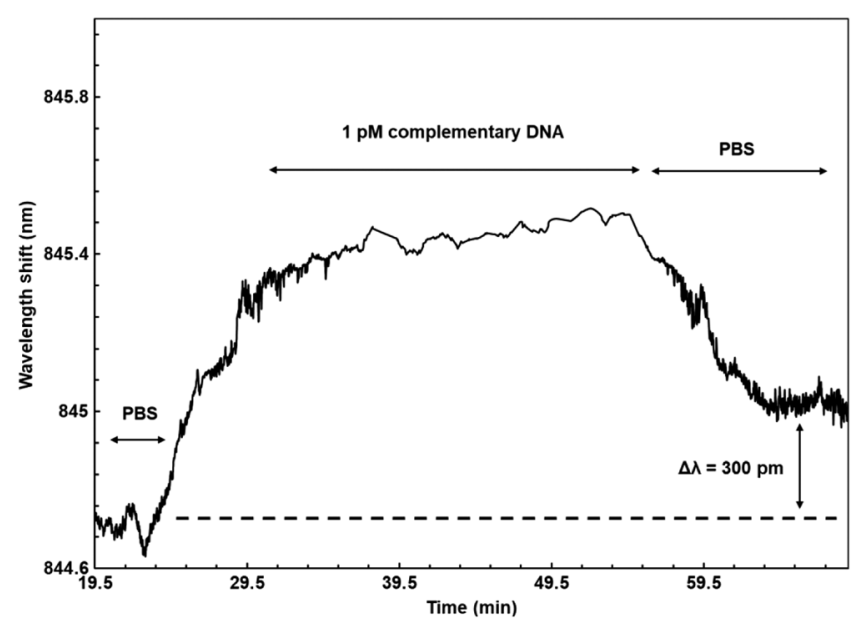

Fig. 3 Wavelength shift due flow of 1 pM complementary DNA on the sensor.

noncomplementary DNA sample was injected onto the sensors at a rate of $10 \mu \mathrm{L} / \mathrm{min}$. The complementary and noncomplementary DNA samples were diluted in the hybridization buffer, which is $1 \mathrm{M} \mathrm{NaCl}, 0.02 \mathrm{M}$ of TrisHCl, and $0.02 \mathrm{M}$ EDTA ( $\mathrm{pH} 7$ ). The measurements were done at a temperature of $30 \mathrm{deg}$. In the figures, there is always a small shift down in signal whenever the DNA samples enter the sensor surface. This is due to the small change in temperature between the sensor surface and the DNA buffer. This lasts for a very short moment. The permanent spectral shift after the second flow of $1 \times$ PBS is stable for $>5 \mathrm{~min}$.

Figure 2 shows the result of flowing 500 pM noncomplementary DNA. Initially, a high spectral shift was encountered due to buffer exchange between PBS and the hybridization buffer as shown in Fig. 2. This high spectral shift was only temporary and started to return back after a while since no binding was occurring between the injected DNA and the immobilized DNA on the sensor. When $1 \times$ PBS was injected afterward, the spectral shift moved back to a very low value (70 pm) as compared with the initial shifted value. The spectral shift did not completely return back to its starting value and this can be attributed to 


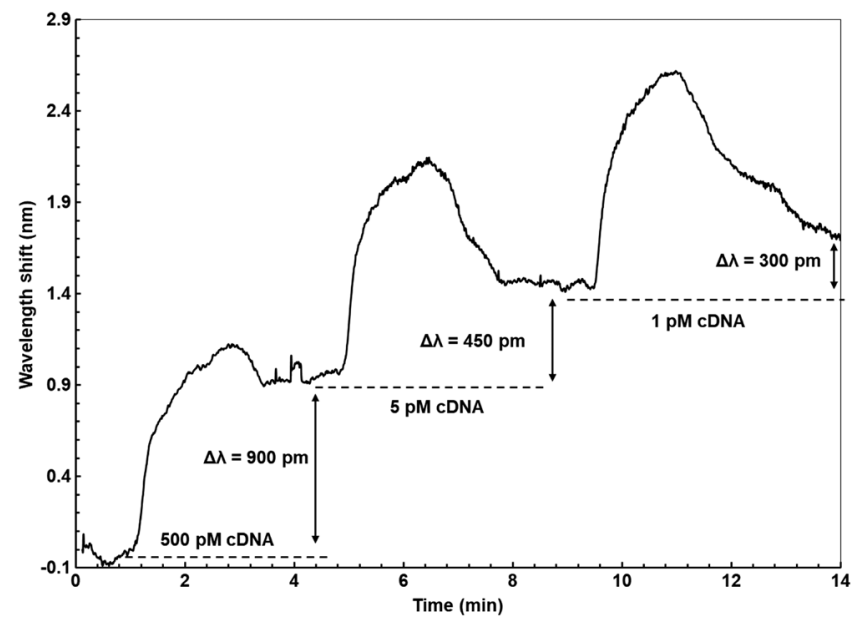

Fig. 4 Wavelength shift due to flow of different concentrations of complementary DNA on the sensor.

nonspecific binding of the noncomplementary DNA samples to the sensor's surface.

Figure 3 shows the case of a 1 pM complementary DNA sample. Again, initially a high increase in the spectral shift was encountered due to the buffer effect. However, afterward, when the $1 \times$ PBS was injected, a high permanent spectral shift compared with its initial value was encountered. This is due to the fact that complementary DNA efficiently bound to the ssDNA immobilized on the sensor surface. The $1 \mathrm{pM}$ complementary DNA sample caused a permanent spectral shift of about $300 \mathrm{pm}$, which is significantly above our detection limit.

Moreover, in Figs. 2, 3, and 6 it looks like there are missing data points. Actually, a new sample was obtained every $0.5 \mathrm{~s}$, but sometimes samples get lost because the data processing does not converge. We, therefore, remade Figs. 2, 3, and 6 to have the same connected line style as Fig. 4, and used a moving average of six data points to smoothen the curve.

Afterward, the spectral shift of a series of different concentrations of complementary DNA was studied. Figure 4 shows the spectral shift due to the injection of 1,5 , and $500 \mathrm{pM}$ of complementary DNA. Initially, the $500 \mathrm{pM}$ complementary DNA was introduced to the chip followed by lower concentrations of 5 and 1 pM complementary DNAs, respectively. As a result, wavelength shifts of 900, 450, and 300 pm were encountered. All of these results are shown in Table 2. The aim to start with high concentration and go down to lower concentrations is to ensure that lower concentrations can still be detected despite

Table 2 Summary of the spectral shift due to different DNA concentrations flown on the sensor surface.

\begin{tabular}{lcc}
\hline DNA type & $\begin{array}{r}\text { DNA concentration } \\
(\mathrm{pM})\end{array}$ & $\begin{array}{c}\text { Wavelength } \\
\text { shift (pm) }\end{array}$ \\
\hline Noncomplementary & 500 & 70 \\
Complementary & 1 & 316 \\
Complementary & 1 & 300 \\
Complementary & 5 & 450 \\
Complementary & 500 & 900 \\
\hline
\end{tabular}

the fact that most of the probe DNA sites were occupied by the initial high concentration targets. The lack of linearity can be due to electrostatic nonspecific binding of the target DNA to the surface of the chip. Additionally, the fact that the sensor surface is not regenerated between concentrations will contribute to the nonlinear response as well.

A dual-mode injector is used to load the complementary DNA samples to be tested in line with the neutral buffer. The injector was loaded manually and the switch of the valve was also done manually. The moment when the valve was switched manually has the first red shift response as shown in Fig. 4 due to the sudden vibration of the fluid delivery tube and then it draws back to normal behavior.

After the proof-of-principle experiments, we moved from spiked artificial DNA samples to samples where the DNA was obtained after a rolling circle amplification (RCA-DNA) and spiked in a realistic amplification buffer. The RCA-DNA obtained the rolling circle amplification process has a concentration of 60 and $100 \mathrm{nM}$. They were used as they obtained without any further dilution to mimic the real clinical process. This buffer is $1 \mathrm{M}$ of $\mathrm{NaCl}, 0.02 \mathrm{M}$ of TrisHCl (pH8), 0.02M of EDTA (pH8), and $0.1 \%$ of Tween-20. The reference buffer for these experiments was $1 \mathrm{M}$ of $\mathrm{NaCl}, 0.02 \mathrm{M}$ of $\mathrm{TrisHCl}(\mathrm{pH} 8)$, and $0.02 \mathrm{M}$ of EDTA (pH8), which is similar to the one in which DNA was prepared except that it does not contain $0.1 \%$ of Tween-20. First, a test on the buffer effect to the signal was done. As shown in Fig. 5, the buffer exchange has no effect on the wavelength spectrum due to the fact that the buffers are identical except for the presence of little amount of tween in the monomer buffer. The temporary increase in the wavelength is due to the presence of the tween in the monomer buffer. Moreover, the signal increased temporarily and then started to return back to its initial value even before the flow of the initial hybridization buffer has started. When the hybridization buffer was injected again, the signal returned back to its initial starting value.

Afterward, a $60 \mathrm{nM}$ of the RCA-DNA samples, which were obtained from the rolling circle amplification process, was injected on the sensor chip and caused a wavelength shift of $400 \mathrm{pm}$ as shown in Fig. 6. A 100-nM RCA-DNA monomer was injected on the sensor chip in a second experiment and a 650-pm wavelength was encountered (data not shown).

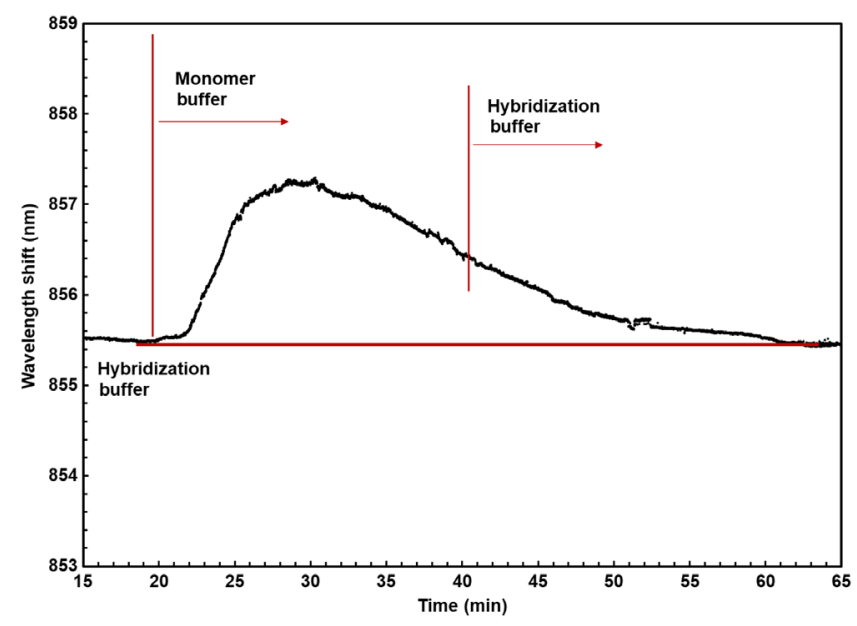

Fig. 5 Wavelength shift due to flow of different buffers. 


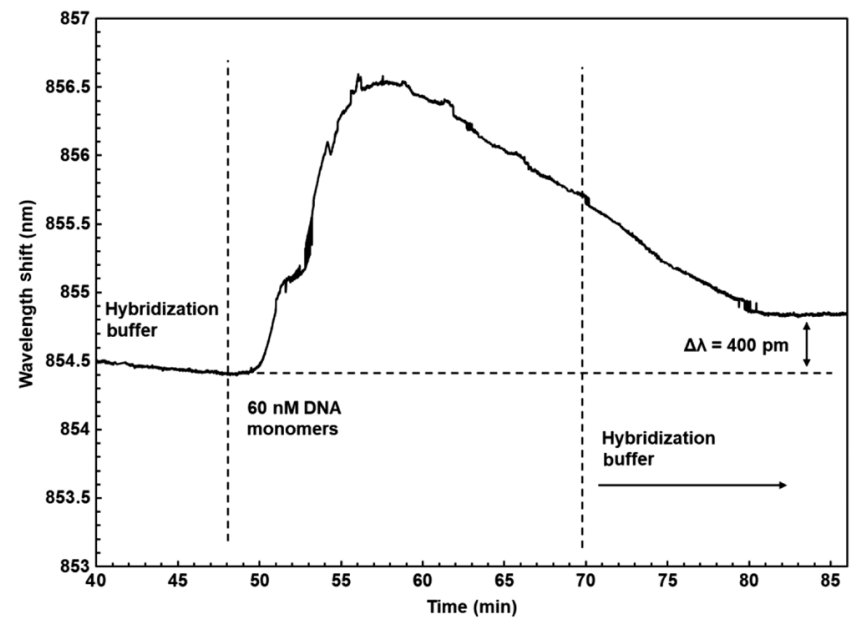

Fig. 6 Wavelength shift due to flow of $60 \mathrm{nM}$ complementary DNA monomers.

\section{Conclusion}

A silicon nitride integrated unbalanced MZI with integrated onchip spectral readout has been investigated in the context of DNA sensing. A 1-pM cDNA encountered a wavelength shift of $300 \mathrm{pm}$, which gives the probability of sensing down to order of 100-fM cDNA. Moreover, RCA-DNA were used on the sensor and preliminary results showed to be promising for real clinical applications. Also, an alternative route to modify the sensor surface with carboxylic groups using the photochemical reaction of fatty acids was proposed and preliminary XPS results were presented.

\section{Disclosures}

The authors have no relevant financial interests in this article and no potential conflicts of interest to disclose.

\section{Acknowledgments}

This work was performed in the context of the EU FP7 project CanDo (grant 610472). We acknowledge Navya Laxman and Mats Nilsson for the DNA products.

\section{References}

1. N. Lagos and M. M. Sigalas, "Single particle detection in a system of two microdisks," Sens. Actuators B 153, 252-255 (2011).

2. S. Zlatanovic et al., "Photonic crystal microcavity sensor for ultracompact monitoring of reaction kinetics and protein concentration," Sens. Actuators B 141, 13-19 (2009).

3. J. Wu and M. Gu, "Microfluidic sensing: state of the art fabrication and detection techniques," J. Biomed. Opt. 16, 080901 (2011).

4. T. Strick et al., "Twisting and stretching single DNA molecules," Prog. Biophys. Mol. Biol. 74, 115-140 (2000).

5. U. Bockelmann et al., "Unzipping DNA with optical tweezers: high sequence sensitivity and force flips," Biophys. J. 82, 1537-1553 (2002).

6. B. van Grinsven et al., "Rapid assessment of the stability of DNA duplexes by impedimetric real-time monitoring of chemically induced denaturation," Lab Chip 11, 1656-1663 (2011).

7. Q. Wang et al., "Non-enzymatic glucose sensing on long and short diamond nanowire electrodes," Electrochem. Commun. 34, 286-290 (2013).

8. J. M. Nam, C. S. Thaxton, and C. A. Mirkin, "Nanoparticle-based biobar codes for the ultrasensitive detection of proteins," Science 301, 1884-1886 (2003).

9. W. S. Yeo et al., "Label-free detection of protein-protein interactions on biochips," Angew. Chem. Int. Ed. 44, 5480-5483 (2005).
10. T. P. Burg et al., "Weighing of biomolecules, single cells and single nanoparticles in fluid," Nature 446, 1066-1069 (2007).

11. A. Poghossian et al., "Field-effect sensors with charged macromolecules: characterisation by capacitance-voltage, constant-capacitance, impedance spectroscopy and atomic-force microscopy methods," Biosens. Bioelectron. 22, 2100-2107 (2007).

12. S. Ingebrandt et al., "Label-free detection of single nucleotide polymorphisms utilizing the differential transfer function of field-effect transistors," Biosens. Bioelectron. 22, 2834-2840 (2007).

13. D. Dey and T. Goswami, "Optical biosensors: a revolution towards quantum nanoscale electronics device fabrication," J. Biomed. Biotechnol. 2011, 1-7 (2011).

14. R. Narayanaswamy and O. S. Wolfbeis, Eds., Optical Sensors, Industrial, Environmental, and Diagnostic Applications, SpringerVerlag, Berlin (2004).

15. A. Serpengüzel, S. Arnold, and G. Griffel, "Excitation of resonances of microspheres on an optical fiber," Opt. Lett. 20(7), 654-656 (1995).

16. A. L. Huston and J. D. Eversole, "Strain-sensitive elastic scattering from cylinders," Opt. Lett. 18(13), 1104-1106 (1993).

17. S. Arnold et al., "Shift of whispering gallery modes in microspheres by protein adsorption," Opt. Lett. 28(4), 272-274 (2003).

18. F. Vollmer et al., "Protein detection by optical shift of a resonant microcavity," Appl. Phys. Lett. 80(21), 4057-4059 (2002).

19. M. A. Santiago-Cordoba et al., "Nanoparticle-based protein detection by optical shift of a resonant microcavity," Appl. Phys. Lett. 99, 073701 (2011).

20. X. D. Fan et al., "Sensitive optical biosensors for unlabeled targets: a review," Anal. Chim. Acta 620, 8-26 (2008).

21. J. L. Nadeau et al., "High-Q whispering-gallery mode sensor in liquids," Proc. SPIE 4629, 172-180 (2002).

22. J. D. Suter et al., "Label-free quantitative DNA detection using the liquid core optical ring resonator," Biosens. Bioelectron. 23, 10031009 (2008).

23. Z. B. Bahşi et al., "A novel label-free optical biosensor using synthetic oligonucleotides from E. coli O157:H7: Elementary sensitivity tests," Sensors 9, 4890-4900 (2009).

24. M. Baaske and F. Vollmer, "Optical resonator biosensors: molecular diagnostic and nanoparticle detection on an integrated platform," ChemPhysChem 13, 427-436 (2012).

25. V. M. N. Passaro et al., "Guided-wave optical biosensors," Sensors 7(4), 508-536 (2007).

26. J. P. Golden et al., "An evanescent-wave biosensor. II. Fluorescent signal acquisition from tapered fiber optic probes," IEEE Trans. Biomed. Eng. 41(6), 585-591 (1994).

27. S. B. Carvalho et al., "A detection and quantification label-free tool to speed up downstream processing of model mucins," PLoS One 13(1), e0190974 (2018).

28. A. Q. Liu et al., "Label-free detection with micro optical fluidic systems (MOFS): a review," Anal. Bioanal. Chem. 391(7), 2443-2452 (2008).

29. P. M. Levine et al., "Real-time, multiplexed electrochemical DNA detection using an active complementary metal-oxide-semiconductor biosensor array with integrated sensor electronics," Biosens. Bioelectron. 24(7), 1995-2001 (2009).

30. F. Vollmer et al., "Multiplexed DNA quantification by spectroscopic shift of two microsphere cavities," Biophys. J. 85(3), 1974-1979 (2003).

31. F. Vollmer and S. Arnold, "Whispering-gallery-mode biosensing: label free detection down to single molecules," Nat. Methods 5(7), 591-596 (2008).

32. K. De Vos et al., "Silicon-on-insulator microring resonator for sensitive and label-free biosensing," Opt. Express 15(12), 7610-7615 (2007).

33. C. Lerma Arce et al., "SOI microring resonator sensor integrated on a fiber facet," in Lab-on-Fiber Technology, Springer Series in Surface Sciences, A. Cusano et al., Eds., vol. 56, Springer, Cham (2015).

34. S. Werquin, Y. De Koninck, and P. Bienstman, "Ring resonators with vertically coupling grating for densely multiplexed applications," IEEE Photonics Technol. Lett. 27, 97-100 (2015).

35. R. Kirchner et al., "Chemical functional polymers for direct UV assisted nanoimprinting of polymeric photonic microring resonators," Phys. Status Solidi A 208(6), 1308-1314 (2011).

36. K. De Vos et al., "SOI optical microring resonator with poly(ethyleneglycol) polymer brush for label-free biosensor applications," Biosens. Bioelectron. 24(8), 2528-2533 (2009). 
37. D. Martens et al., "Compact silicon nitride arrayed waveguide gratings for very near-infrared wavelengths," IEEE Photonics Technol. Lett. 27(2), 137-140 (2015).

38. R. Stine et al., "Formation of primary amines on silicon nitride surfaces: a direct, plasma-based pathway to functionalization," Langmuir 23, 4400-4404 (2007).

39. A. F. Gavela et al., "Last advances in silicon-based optical biosensors," Sensors 16, 285 (2016).

40. E. T. Vandenberg et al., "Structure of 3-aminopropyl triethoxy silane on silicon-oxide," J. Colloid Interface Sci. 147(1), 103-118 (1991).

41. K. van der Maaden et al., "Fluorescent nanoparticle adhesion assay: a novel method for surface $\mathrm{pK}(\mathrm{a})$ determination of self-assembled monolayers on silicon surfaces," Langmuir 28(7), 3403-3411 (2012).

42. M. S. Murib et al., "Heat-transfer based characterization of DNA on synthetic sapphire chips," Sens. Actuators B Chem. 230, 260-271 (2016).

43. I. M. Klotz, "Succinylation," in Methods in Enzymology, C. H. W. Hirs, ed., vol. 11, p. 576, Academic Press, New York (1967).

44. V. Vermeeren et al., "Topographical and functional characterization of the ssdna probe layer generated through EDC mediated covalent attachment to nanocrystalline diamond using fluorescence microscopy," Langmuir 24, 9125-9134 (2008).

45. P. Christiaens et al., "EDC-mediated DNA attachment to nanocrystalline CVD diamond films," Biosens. Bioelectron. 22, 170-177 (2006).

46. W. Yang et al., "DNA-modified nanocrystalline diamond thin-films as stable, biologically active substrates," Nat. Mater. 1, 253-257 (2002).

47. R. Alemany Server et al., "Advances towards reliable identification and concentration determination of rare cells in peripheral blood," Proc. SPIE 9705, 97050G (2016).

48. D. Martens and P. Bienstman, "Comparison between Vernier-cascade and MZI as transducer for biosensing with on-chip spectral filter," Nanophotonics 6, 703-712 (2017).
49. A. Z. Subramanian et al., "Low-loss single mode PECVD silicon nitride photonic wire waveguides for 532-900 nm wavelength window fabricated within a CMOS pilot line," IEEE Photonics J. 5, 2202809 (2013).

50. D. Martens et al., "Low-cost integrated biosensing platform based on SiN nanophotonics for biomarker detection in urine," Anal. Methods 10, 3066-3073 (2018).

Mohammed Sharif Murib received his BSc degree in physics from Lebanese University, Faculty of Science, Hadath, in 2005. He received his MSc degree in physics from Koç University in 2009. He received his $\mathrm{PhD}$ in February 2014. During his MSc and PhD degrees programs he worked on optoelectronics, bioelectronics, and biophotonic sensors. Afterwards, Joined UGent/IMEC, Department of Information Technology (INTEC) of Ghent University as a researcher. Currently, he is at ASML as optical design engineer.

Daan Martens received his master's degree in engineering physics in 2012 and his PhD from Ghent University in 2017. His current research interests include integrated photonic biosensors, the VNIR wavelength range, silicon nitride, and on-chip spectral filters. He worked in the Photonics Research Group at Ghent University from 2012 till 2018.

Peter Bienstman received his degree in electrical engineering from Ghent University, Belgium, in 1997 and his PhD from the same university in 2001, at the Department of Information Technology (INTEC), where he is currently working as an associate professor. During 2001 to 2002 , he spent a year in the Joannopoulos research group at MIT. His research interests include several applications of nanophotonics (including biosensors and photonic information processing) as well as nanophotonics modeling. He has published over 110 papers and holds several patents. He has been awarded a ERC starting grant for the Naresco-project: novel paradigms for massively parallel nanophotonic information processing. 